\title{
NOISE REDUCTION RESULTS OF THE ACASIAS ACTIVE LINING PANEL
}

\section{EMUS 2020}

\author{
M. MISOL*, S. ALGERMISSEN* \\ * German Aerospace Center (DLR) \\ Institute of Composite Structures and Adaptive Systems, Braunschweig, Germany \\ e-mail: malte.misol@dlr.de,web page: www.dlr.de/fa/en
}

Key words: Active Noise Reduction, ANC, ASAC, Interior Noise, Aircraft, CROR

\begin{abstract}
Advanced concepts for aero-structures with multifunctional capabilities are investigated within the EU-project ACASIAS. In work package 3 of ACASIAS, components of an active noise reduction system are structurally integrated into a curved sandwich panel by means of 3D printed inserts. This so-called smart lining is intended for application in aircraft as a modular and lightweight interior noise treatment in propeller-driven aircraft. The broad application scenario of smart linings ranges from retro-fitting of current regional aircraft such as ATR 42, ATR 72, DHC-8 Q400 to the application in new short-range aircraft with energy efficient counter rotating open rotor (CROR) engines or with distributed electric propellers. A key feature of the smart lining with integrated active components is its modularity, facilitating a flexible application in the aircraft cabin. This requires a fully self-contained sensing mechanism based on structurally integrated accelerometers. Using the normal surface vibration data from the integrated sensors, the smart lining is able to predict the sound field in front of it. The so-called virtual microphone method with remote sensors and observer filter allows to get rid of real microphones and wiring in the aircraft cabin. This makes retro-fitting easier because it reduces wiring effort and costs which is beneficial for future aircraft as well. However, the use of virtual instead of real microphones might deteriorate the performance or even the stability of the active noise reduction system because it relies on accurate plant models. Laboratory experiments in a sound transmission loss facility are conducted to assess the behavior of the smart lining with virtual microphones and compare it to a smart lining with real microphones. The sensitivity of the smart lining to environmental changes and the noise reduction performance and control system stability are investigated in this study.
\end{abstract}

\section{INTRODUCTION}

Active noise control is able to reduce cabin noise in propeller driven aircraft. Different approaches are pursued since the late 1980s. One approach followed by Elliott et al. [1] uses loudspeakers to generate anti-sound which destructively interferes with the cabin noise. An alternative approach is the active structural acoustic control (ASAC). The ASAC method requires structural actuators and sensors to control the sound radiating structural vibration of surfaces. 
Early results of ASAC are published by Fuller and Jones [2]. One realization of an ASAC system uses actuators and sensors applied to the sidewall panels (linings). Early experiments with such active linings are documented in Lyle and Silcox [3]. Active linings with electrodynamic exciters as actuators are successfully realized by Misol et al. [4] and by Misol [5]. In [4] tests of an active lining in a sound transmission loss facility and in [5], full-scale tests of two active lining modules mounted in the cabin of a Dornier Do728 aircraft are reported.

The active noise control systems mentioned so far have in common that they use microphones as error sensors. However, the requirement of having distributed and closely adjacent microphones in the whole cabin is undesirable because it requires additional wiring and prevents flexible cabin layouts. The so-called smart lining concept proposed by Misol et al. [4] tries to overcome these drawbacks by modular active linings with structurally integrated actuators, sensors and control. This concent requires a substitution of the phvsical error microphones by virtual error microphones. One applicable method is the remote microphone technique for active control proposed by Roure and Albarazzin [6]. In this technique the error microphones are substituted by remote microphones and an observer filter. In a similar approach, Cheer and Daley [7] replace the remote microphones by accelerometers mounted on the radiating structure. This approach is adopted for the smart lining concept.

The present contribution focuses on the performance and robustness of the ACASIAS active

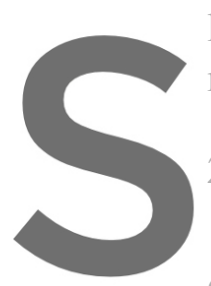
lining panel in the case

ment data and identified

2 Experiments

The experiments are done
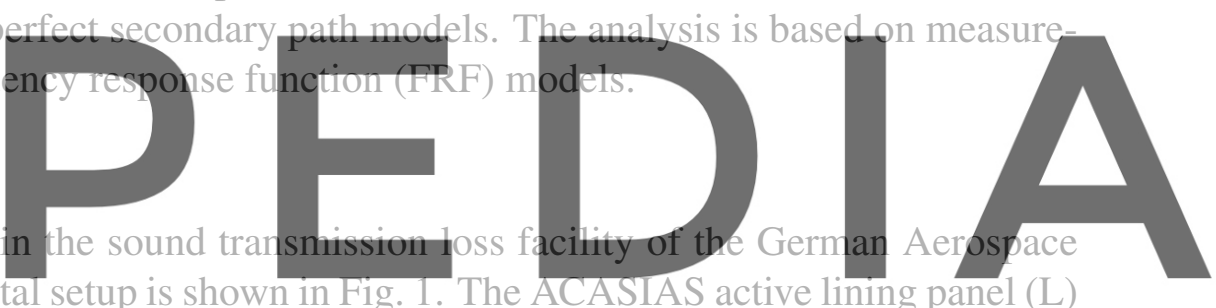

Center (DLR). The experimental setup is shown in Fig. 1. The ACASIAS active lining panel (L)

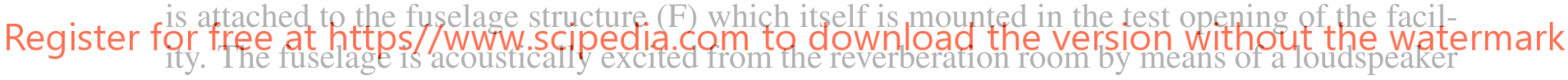
array. The excitation sound field is typical for a counter rotating open rotor (CROR) engine. It contains the first five harmonics at $119.4 \mathrm{~Hz}, 149.2 \mathrm{~Hz}, 268.6 \mathrm{~Hz}, 387.5 \mathrm{~Hz}$ and $417.9 \mathrm{~Hz}$. The transmitted sound is measured in the semi-anechoic room by means of a microphone array with 24 microphones. Measurements are repeated for ten different distances between microphone plane and lining. The reference signal $x$ is used to assemble the signals from the sequential measurements correctly. The hardware used for data sampling and real-time control is a MicroLabBox from dSPACE (DSP). The sampling rate is set to $2000 \mathrm{~Hz}$. All analog input and output signals are bandlimited to $500 \mathrm{~Hz}$ using low-pass filters (LPF). The control signals are amplified with a power amplifier (AMP). A detailed description of the smart lining and the actuator and sensor locations is given in Algermissen and Misol. [8]. The signals from the accelerometers $\mathbf{d}_{\mathbf{s}}$, microphones $\mathbf{d}_{\mathbf{a}}$ and the reference signal $x$ are used as inputs for the control plant shown in Fig. 2.

\section{Simulations}

A block diagram of the control plant is shown in Figure 2. The green blocks are input signals and the blue blocks are FRF models both obtained from experiments. The grey blocks perform 


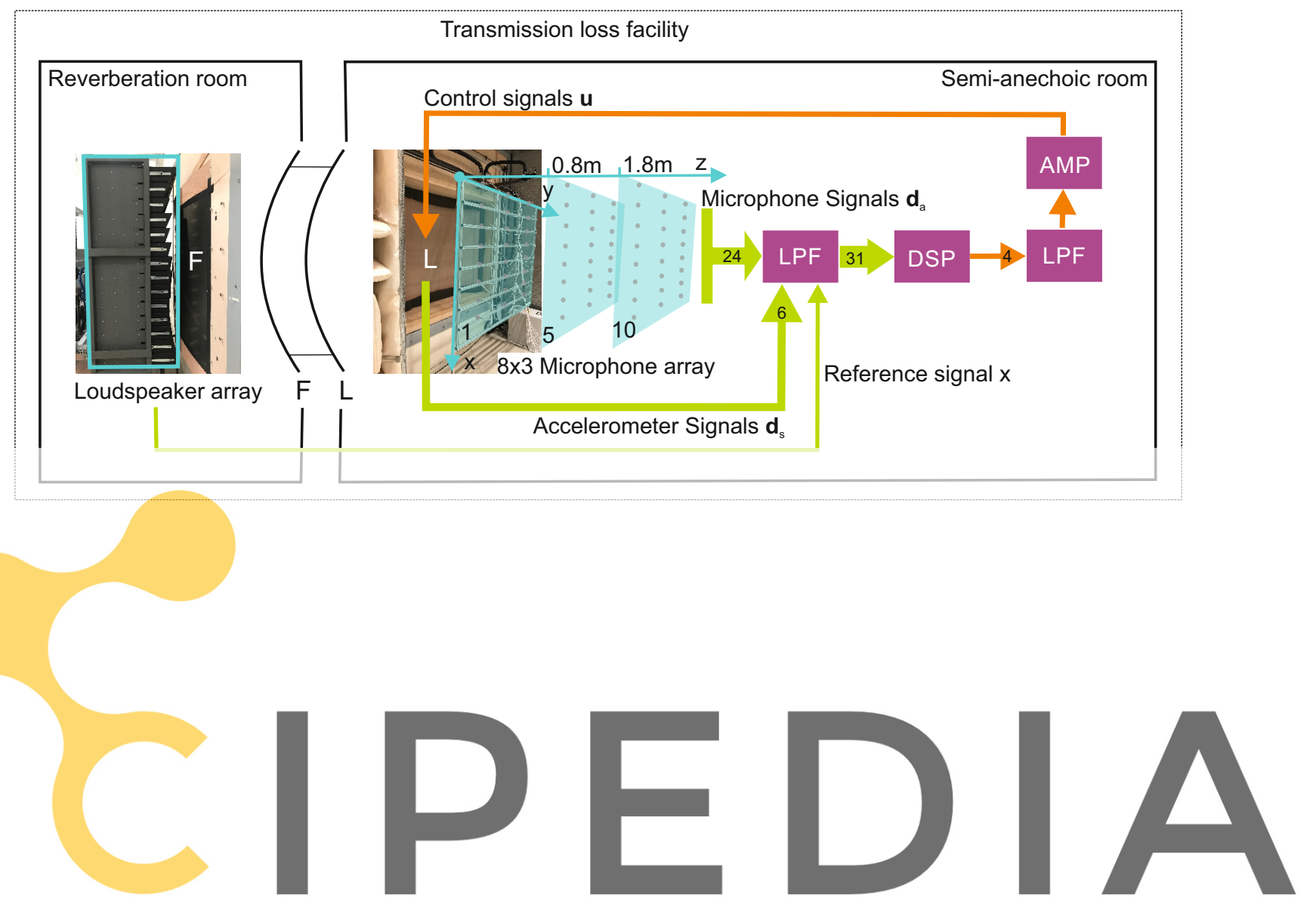

Register for free at https//www.scipedia.com to download the version without the watermark 


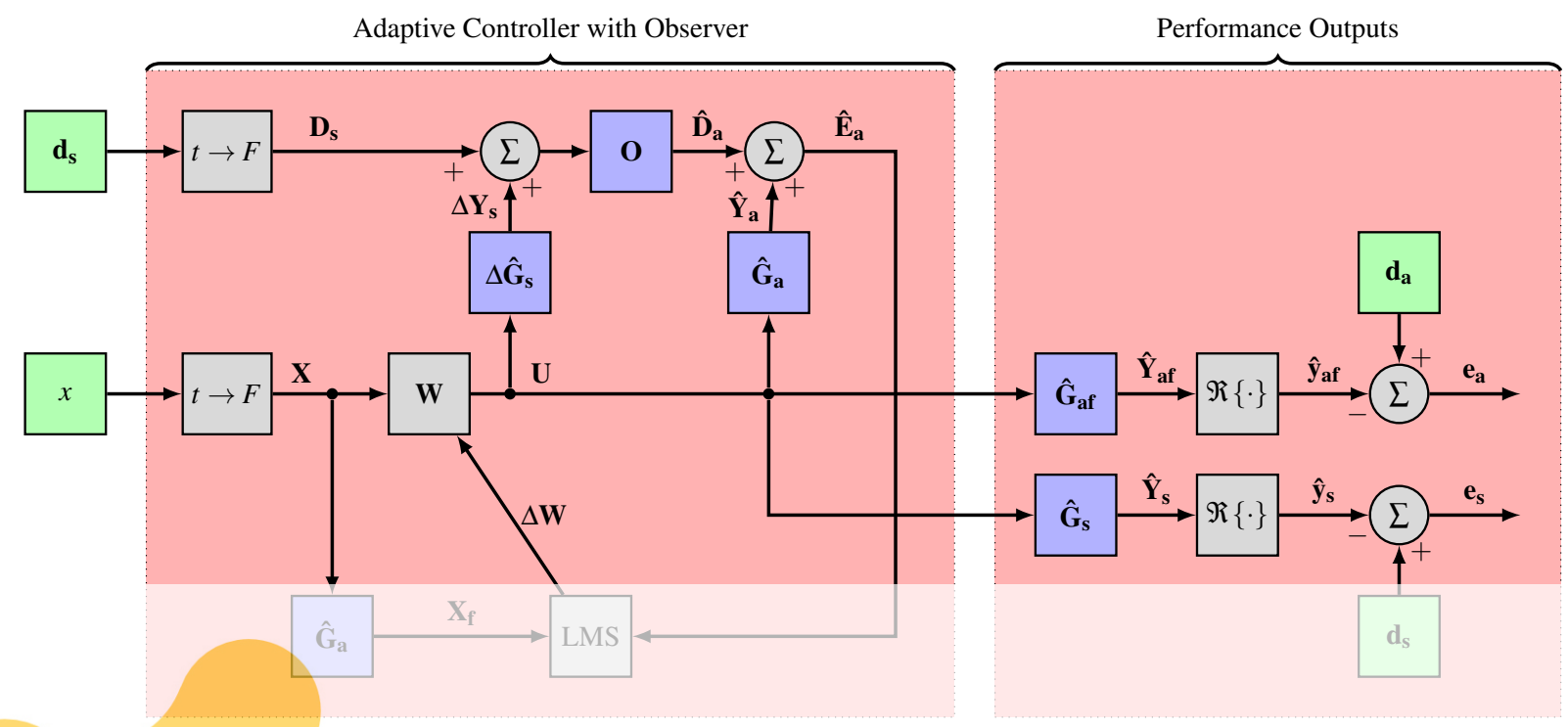

Figure 2: Block diagram of the control plant.

performance. It will be shown in the following Section that a nonzero $\beta$ is required to stabilize certain harmonics if

4 Results

Figure 3 shows the sound prestis

and 10 (see Fig.
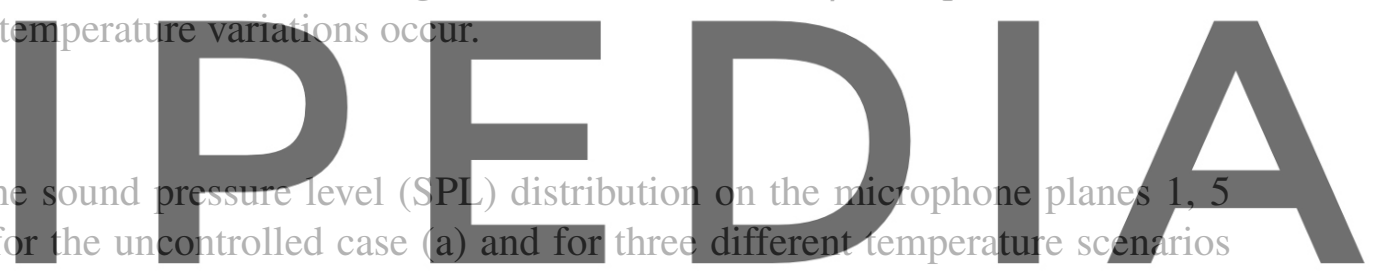

(b), (c) and (d). The other microphone planes are omitted for reasons of clarity. This is possible

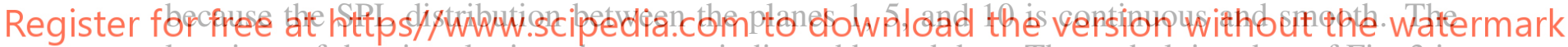
locations of the virtual microphones are indicated by red dots. The underlying data of Fig. 3 is from the performance output $\mathbf{e}_{\mathbf{a}}$ in Fig. 2. The sound pressure reductions are calculated relative to the measured disturbance sound pressures $\mathbf{d}_{\mathbf{a}}$. In the uncontrolled case (a), a decrease of the sound pressure level can be seen with increasing distance $(z)$ from the lining. Scenario (b) represents the ideal control scenario with $\Delta \hat{\mathbf{G}}_{\mathbf{s}}=0$. This means either constant temperature conditions or perfect (temperature dependent) secondary path modeling. In this scenario a mean SPL reduction of $10 \mathrm{~dB}$ and $5.9 \mathrm{~dB}(\mathrm{~A})$ is achieved on plane $1(z=0)$ and a mean SPL reduction of $8 \mathrm{~dB}$ and $5.7 \mathrm{~dB}(\mathrm{~A})$ is achieved on planes 1-10 (240 virtual microphones). In scenario (c) it is assumed that the structural secondary path model $\hat{\mathbf{G}}_{\mathbf{s}}{ }^{T_{2}}$ is identified for $T_{2}=30^{\circ} \mathrm{C}$ and the temperature during real-time control is $T_{1}=22^{\circ} \mathrm{C}$ (or vice versa). This means $\Delta \hat{\mathbf{G}}_{\mathbf{s}} \neq 0$ corresponding to an imperfect compensation of the actuator feedback on the remote sensors (accelerometers). In this scenario all eigenvalues $\lambda$ are positive, but the smallest eigenvalue associated with the frequency of the second harmonic is close to zero and must be stabilized by taking $\beta=0.0366$. The implications on control performance are visible in Fig. 3 (c). A mean SPL reduction of $4.5 \mathrm{~dB}$ and $3.2 \mathrm{~dB}(\mathrm{~A})$ is achieved on plane $1(z=0)$ and a mean SPL reduction of $4.8 \mathrm{~dB}$ and $3.9 \mathrm{~dB}(\mathrm{~A})$ is achieved on planes 1-10. A further degradation of control performance occurs in scenario (d) where it is assumed that the structural secondary path model 
(a)

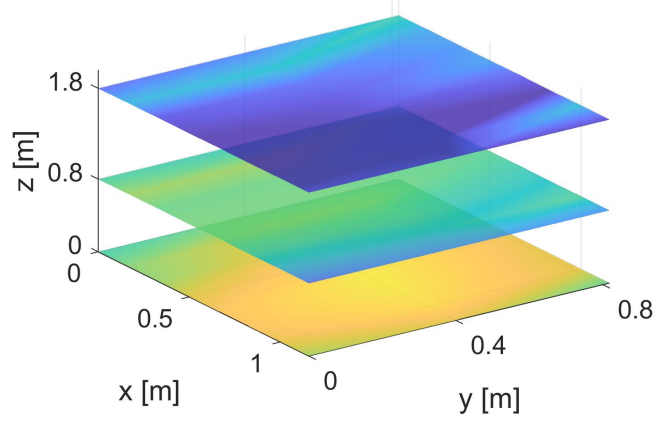

(b)

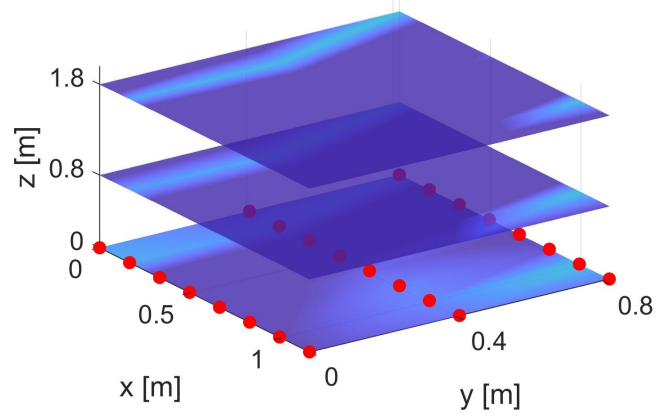

(d)

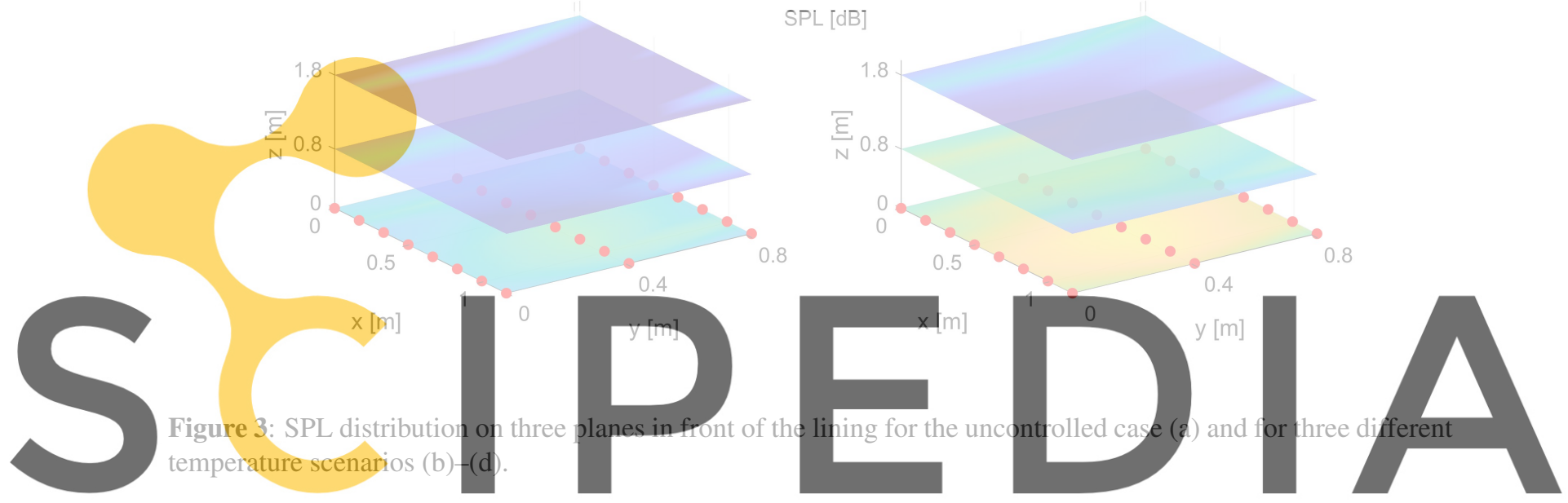

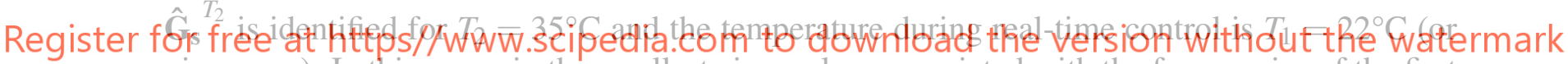
vice versa). In this scenario the smallest eigenvalues associated with the frequencies of the first and the second harmonic are negative and must be stabilized by taking $\beta=3.2764$ for the first and $\beta=2.0947$ for the second harmonic. Such strong control weighting implies that the SPL at the first two harmonics will not be affected by the active controller. Since these two harmonics dominate the SPL, Fig. 3 (a) and (d) are very similar. In scenario (d) a mean SPL reduction of $0.14 \mathrm{~dB}$ and $0.13 \mathrm{~dB}(\mathrm{~A})$ is achieved on plane $1(z=0)$ and a mean SPL reduction of $0.11 \mathrm{~dB}$ and $0.013 \mathrm{~dB}(\mathrm{~A})$ is achieved on planes $1-10$. The results clearly underline that a temperature compensation of the secondary path model is useful and might be necessary. However, it is unclear how much the temperature of the lining actually varies during flight since, as an interior part, it is thermally coupled to the cabin and isolated from the fuselage by an air gap filled with glass fiber insulation bags. Furthermore, the variation of the acoustic secondary path $\mathbf{G}_{\mathbf{a}}$ due to changes in temperature, seat occupation and other factors will have a negative influence on the noise reduction performance as well. But it will not affect the stability of the control system since the acoustic secondary path model $\hat{\mathbf{G}}_{\mathbf{a}}$ is an integral part of the adaptive controller with virtual microphones (see Fig. 2). It remains a future task to assess the implications of imperfect acoustic secondary path models on the control performance. 


\section{ACKNOWLEDGEMENTS}

${ }_{* * * *}$ This project has received funding from the European Union's Horizon 2020 research and innovation programme under grant agreement No. 723167.

The authors gratefully acknowledge the support of Florian Hesselbach, Alexander Rehmann, Markus Klingseis and Dr. Dietmar Völkle from DIEHL Aviation Laupheim.

\section{REFERENCES}

[1] S. J. Elliott, P. A. Nelson, I. M. Stothers, and C. C. Boucher, "In-flight experiments on the active control of propeller-induced cabin noise," Journal of Sound and Vibration, vol. 140, no. 2, pp. 219-238, 1990.

[2] C. R. Fuller and J. D. Jones, "Experiments on reduction of propeller induced interior noise by active control of cylinder vibration," Journal of Sound and Vibration, vol. 112, no. 2, pp. 389-395, Jan. 1987.

[3] K. H. Lyle and R. J. Silcox, "A study of active trim panels for interior noise reduction in an aircraft fuselage," in SAE Technical Paper. SAE International, 05 1995. [Online]. Available: https://doi.org/10.4271/951179
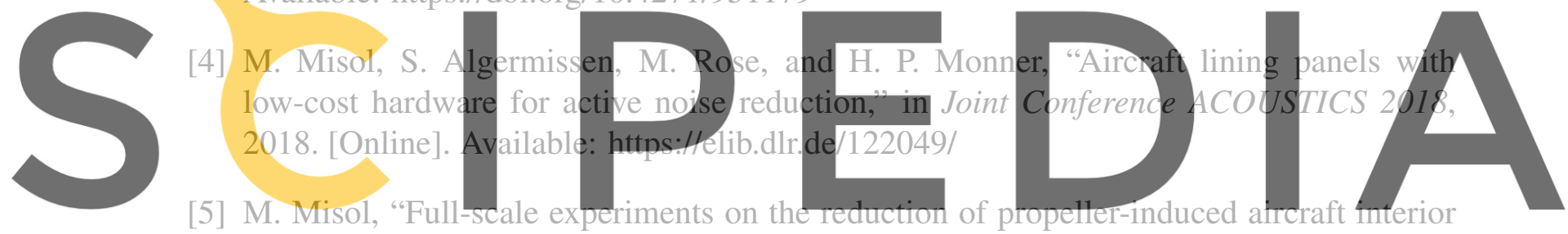
noise with active trim panels." Applied Acoustics, vol. 159, p. 107086, 2020. [Online].

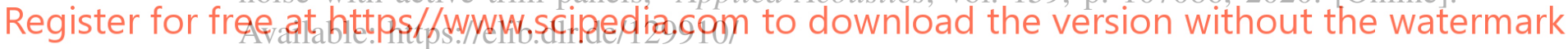

[6] A. Roure and A. Albarrazin, "The remote microphone technique for active noise control," in PROCEEDINGS OF ACTIVE 99: THE INTERNATIONAL SYMPOSIUM ON ACTIVE CONTROL OF SOUND AND VIBRATION, VOLS 1 \& 2, 1999, pp. 1233-1244.

[7] J. Cheer and S. Daley, "Active structural acoustic control using the remote sensor method," Journal of Physics: Conference Series, vol. 744, no. 1, 2016.

[8] S. Algermissen and M. Misol, "Experimental Analysis of the ACASIAS Active Lining Panel," in Proc. of European Conference on Multifunctional Structures (EMuS), X. Martinez and H. Schippers, Eds., 2020, online event, November 17-18.

[9] M. Misol, "Active Sidewall Panels with Virtual Microphones for Aircraft Interior Noise Reduction," Applied Sciences, vol. 10, no. 6828, pp. 1-13, 2020. [Online]. Available: https://elib.dlr.de/136353/

[10] S. J. Elliott, Signal Processing for Active Control. London: Academic Press, 2001. 